\title{
ISOMORPHISMS OF GRAPH GROUPS
}

\author{
CARL DROMS
}

\begin{abstract}
Given a graph $X$, define the presentation $P X$ to have generators the vertices of $X$, and a relation $x y=y x$ for each pair $x, y$ of adjacent vertices. Let $G X$ be the group with presentation $P X$, and given a field $K$, let $K X$ denote the $K$-algebra with presentation $P X$. Given graphs $X$ and $Y$ and a field $K$, it is known that the algebras $K X$ and $K Y$ are isomorphic if and only if the graphs $X$ and $Y$ are isomorphic. In this paper, we use this fact to prove that if the groups $G X$ and $G Y$ are isomorphic, then so are the graphs $X$ and $Y$.
\end{abstract}

Given a graph $X$ with vertex set $V(X)$, we define the presentation $P X$ to be that having as generators the elements of $V(X)$, with a defining relation $v w=w v$ for each pair $v$ and $w$ of adjacent vertices of $X . P X$ can be regarded as a presentation of a group $G X$, or of a $K$-algebra $K X$ over a field $K$. In [1] , Kim, Makar-Limanov, Neggers, and Roush proved that if the algebras $K X$ and $K Y$ are isomorphic, then so are the graphs $X$ and $Y$. (In their formulation, two generators commute provided they are not adjacent in the graph. This is sufficient for our purposes, since if two graphs are isomorphic, so are their complements.)

Let $K$ be a field. In this note we will show that if the groups $G X$ and $G Y$ are isomorphic, then so are the algebras $K X$ and $K Y$, thus demonstrating:

THEOREM. If the groups $G X$ and $G Y$ are isomorphic, then so are the graphs $X$ and $Y$.

Let $f: G X \rightarrow G Y$ be an isomorphism. Denote by $G_{2} X$ the quotient group $G X /\left[G X,(G X)^{\prime}\right]$. Then $f$ induces an isomorphism $f_{2}: G_{2} X \rightarrow G_{2} Y$. Let $V(X)$ and $V(Y)$ be totally ordered, and denote both orderings by $<$. We will not distinguish by notation between a vertex of $X$, the corresponding element of $G X$, and the image of this element in $G_{2} X$. For each vertex $x$ of $X, f_{2}(x)$ can be written uniquely in the form $y_{1}^{a_{1}} y_{2}^{a_{2}} \cdots y_{n}^{a_{n}} C_{x}$, where $C_{x}$ is an element of the commutator subgroup $\left(G_{2} Y\right)^{\prime}, y_{1}<y_{2}<\cdots<y_{n}$, and the integers $a_{r}$ are all nonzero. Define $f_{*}(x)=a_{1} y_{1}+a_{2} y_{2}+\cdots+a_{n} y_{n}$. We will show that the function $f_{*}: X \rightarrow K Y$ extends to a homomorphism $f_{*}: K X \rightarrow K Y$ by showing that if $x x^{\prime}=x^{\prime} x$ is a relation of $P X$, then $f_{*}(x) f_{*}\left(x^{\prime}\right)=f_{*}(x) f_{*}\left(x^{\prime}\right)$ in $K Y$.

LEMMA. The commutators $\left\{\left[x_{i}, x_{j}\right] \mid x_{i}<x_{j}\right.$ and $x_{i}$ and $x_{j}$ are not adjacent in $X\}$ of $G_{2} X$ are linearly independent.

PROOF. Consider the exact sequence

$$
1 \rightarrow N \rightarrow F X \rightarrow G X \rightarrow 1
$$

Received by the editors January 21, 1985 and, in revised form, May 12, 1986.

1980 Mathematics Subject Classification (1985 Revision). Primary 20F05; Secondary 20F12. 
associated with the presentation $P X$. Since $N$ is a subgroup of $F X^{\prime}$, there is an exact sequence

$$
1 \rightarrow \hat{N} \rightarrow\left(F_{2} X\right)^{\prime} \rightarrow\left(G_{2} X\right)^{\prime} \rightarrow 1
$$

where $\hat{N}$ denotes the image of $N$ in $F_{2} X$. By the Basis Theorem, $\left(F_{2} X\right)^{\prime}$ is a free abelian group with a basis consisting of the commutators $\left\{\left[x_{i}, x_{j}\right] \mid x_{i}<x_{j}\right\}$. Since $\hat{N}$ is the subgroup of $\left(F_{2} X\right)^{\prime}$ generated by the collection $\left\{\left[x_{i}, x_{j}\right] \mid x_{i}<x_{j}, x_{i}\right.$ and $x_{j}$ adjacent $\}$, the assertion follows.

Suppose that $x$ and $x^{\prime}$ are adjacent vertices of $X$. Let $f_{2}(x)=y_{1}^{a_{1}} y_{2}^{a_{2}} \cdots y_{n}^{a_{n}} C_{x}$ and $f_{2}\left(x^{\prime}\right)=y_{1}^{b_{1}} y_{2}^{b_{2}} \cdots y_{n}^{b_{n}} C_{x^{\prime}}$, with $y_{1}<y_{2}<\cdots<y_{n}$, where we allow some of the exponents $a_{r}$ and $b_{s}$ to be 0 so that we may use the same elements of $V(Y)$ in both expressions. Writing $\left(G_{2} Y\right)^{\prime}$ additively, and recalling that in $G_{2} Y$, commutators are central, we have

$$
\begin{aligned}
0 & =\left[f_{2}(x), f_{2}\left(x^{\prime}\right)\right]=\sum_{r, s}\left[y_{r}^{a_{r}}, y_{s}^{b_{s}}\right] \\
& =\sum_{r, s} a_{r} b_{s}\left[y_{r}, y_{s}\right]=\sum_{r<s}\left(a_{r} b_{s}-a_{s} b_{r}\right)\left[y_{r}, y_{s}\right] .
\end{aligned}
$$

Thus, by the Lemma, if $y_{r}$ and $y_{s}$ are nonadjacent vertices of $Y$, then $a_{r} b_{s}-a_{s} b_{r}=0$. Clearly, $f_{*}(x)=\sum_{r} a_{r} y_{r}$ and $f_{*}\left(x^{\prime}\right)=\sum_{s} b_{s} y_{s}$, so that

$$
f_{*}(x) f_{*}\left(x^{\prime}\right)-f_{*}\left(x^{\prime}\right) f_{*}(x)=\sum_{r, s}\left(a_{r} b_{s}-b_{r} a_{s}\right) y_{r} y_{s}
$$

If $y_{r}$ and $y_{s}$ are adjacent vertices of $Y$, then $y_{r} y_{s}=y_{s} y_{r}$ in $K Y$, so the net coefficient of $y_{r} y_{s}$ in this sum is $\left(a_{r} b_{s}-b_{r} a_{s}\right)+\left(a_{s} b_{r}-b_{s} a_{r}\right)=0$. If $y_{r}$ and $y_{s}$ are nonadjacent vertices of $Y$, then the net coefficient of $y_{r} y_{s}$ is $a_{r} b_{s}-b_{r} a_{s}$, and we saw above that this must by 0 when $y_{r}$ and $y_{s}$ are not adjacent. Finally, for each $r$, the coefficient of $y_{r}^{2}$ is $a_{r} b_{r}-b_{r} a_{r}=0$. Thus, $f_{*}(x) f_{*}\left(x^{\prime}\right)-f_{*}\left(x^{\prime}\right) f_{*}(x)=0$, so $f_{*}$ extends to a homomorphism from $K X$ to $K Y$.

It is now an easy matter to check that for each vertex $x$ of $X,\left(f^{-1}\right)_{*} f_{*}(x)=x$, and for each vertex $y$ of $Y, f_{*}\left(f^{-1}\right)_{*}(y)=y$, so that $f_{*}$ is in fact an isomorphism.

The following shorter proof of our theorem has been pointed out by an anonymous reviewer: Define $G_{0}=G X$ and $G_{n+1}=\left[G_{n}, G\right]$ for $n \geq 0$, and let $L X$ denote the Lie algebra $K \otimes \sum G_{n} / G_{n+1}$. Then the algebra $K X$ is the universal enveloping algebra of $L X$, so that if $G X$ and $G Y$ are isomorphic groups, then the Lie algebras $L X$ and $L Y$, and hence the algebras $K X$ and $K Y$, are isomorphic.

\section{REFERENCES}

1. K. H. Kim, L. Makar-Limanov, J. Neggers and F. Roush, Graph algebras, J. Algebra 64 (1980), 46-51.

Department of Mathematics and Computer Science, James Madison UniverSITY, HARRISONBURG, VIRGINIA 22807 